\title{
Exact Current Statistics of the Asymmetric Simple Exclusion Process with Open Boundaries
}

\author{
Mieke Gorissen, ${ }^{1}$ Alexandre Lazarescu, ${ }^{2}$ Kirone Mallick, ${ }^{2}$ and Carlo Vanderzande ${ }^{1,3}$ \\ ${ }^{1}$ Faculty of Sciences, Hasselt University, 3590 Diepenbeek, Belgium \\ ${ }^{2}$ Institut de Physique Théorique, C. E. A. Saclay, 91191 Gif-sur-Yvette Cedex, France \\ ${ }^{3}$ Instituut Theoretische Fysica, Katholieke Universiteit Leuven, 3001 Heverlee, Belgium
}

(Received 2 August 2012; published 22 October 2012)

\begin{abstract}
Nonequilibrium systems are often characterized by the transport of some quantity at a macroscopic scale, such as, for instance, a current of particles through a wire. The asymmetric simple exclusion process (ASEP) is a paradigm for nonequilibrium transport that is amenable to exact analytical solution. In the present work, we determine the full statistics of the current in the finite size open ASEP for all values of the parameters. Our exact analytical results are checked against numerical calculations using density matrix renormalization group techniques.
\end{abstract}

DOI: 10.1103/PhysRevLett.109.170601

PACS numbers: 05.60.- $\mathrm{k}, 02.50 . \mathrm{Ga}, 05.40 .-\mathrm{a}$

A system containing carriers (of thermal energy, mass, or electrical charge) and subject to a driving field in its bulk, or to a difference of potentials between its boundaries, will usually evolve to a nonequilibrium steady state with a nonvanishing macroscopic current of heat, particles, or charges flowing through it. Because of the presence of this macroscopic current, time-reversal invariance is violated. This is a situation which lies beyond the realm of traditional thermodynamics: Steady-state transitions at the microscopic level break detailed balance, and the principles of equilibrium statistical mechanics do not apply. Hence, for a system that is bulk-driven, boundary-driven, or both, no suitable generalization of the Gibbs-Boltzmann formalism exists that would allow us to predict the value of the current and of its fluctuations from first principles.

During the past two decades, substantial progress has been made towards a statistical theory of nonequilibrium systems [1-6]. Large-deviation functions, that encode atypical fluctuations of a physical observable, are likely to be the best candidates to generalize the traditional thermodynamic potentials. Moreover, it has been proved that large-deviation functions display symmetry properties, called "fluctuation theorems," that remain valid far from equilibrium [2]. These remarkable relations imply linear response theory in the vicinity of equilibrium. Hence, the determination of large deviations in a nonequilibrium system, whether theoretically, numerically, or experimentally, is a question of fundamental importance [7-14].

There are very few models in nonequilibrium physics that can be studied analytically. Among these, the asymmetric simple exclusion process (ASEP) has become a paradigm [15-17]. The ASEP is a one-dimensional lattice gas model in which particles perform biased random walks and interact through an exclusion constraint that mimics a hard-core repulsion: Two particles cannot occupy the same site at a given time. This minimal system appears as a building block in a great variety of phenomena that involve low-dimensional transport with constraints. Invented origi- nally to represent the motion of ribosomes along mRNA during protein synthesis, this model plays a seminal role in nonequilibrium statistical mechanics and has been applied to problems as different as surface growth, biological transport, traffic flow, and pure mathematics [5,17-22].

In the long time limit, the ASEP reaches a nonequilibrium steady state with a fluctuating macroscopic current. Exact results have been derived for the exclusion process on a periodic ring and on the infinite line, using the Bethe ansatz, determinantal processes, and random matrix theory [22-26]. For open boundaries, the steady state has a recursive structure [27] that can be encoded by a matrix product representation [28], a fruitful method to analyze low-dimensional transport models $[5,29]$. The mean value of the stationary current, the associated density profiles, and the phase diagram of the open ASEP are known exactly $[27,28]$. However, finding the full statistical properties of the current in the open ASEP has remained, until now, an outstanding challenge that has stimulated much work [8,11,30-35]. A recent conjecture based on the Bethe ansatz [30] gives the asymptotic behavior of the largedeviation function of the current for infinitely large systems in some specific regions of the phase diagram. In the present work, we give exact analytic expressions for the full statistics of the current that are valid for arbitrary system sizes and boundary parameters, thus solving this long-standing problem.

The dynamics of the ASEP is that of a continuous time Markov chain: During an infinitesimal time interval $d t$, a particle located on a site can jump forward to the next adjacent site with rate 1 and hop backward to the previous site with rate $q$, provided these sites are empty. A particle can enter site 1 with rate $\alpha$ and site $L$ with rate $\delta$ and can exit from site 1 with rate $\gamma$ and from site $L$ with rate $\beta$ (see Fig. 1). Each of the $2^{L}$ microscopic configurations $\mathcal{C}$ of the ASEP can be written as a binary string of length $L$ $\left(\tau_{1}, \ldots, \tau_{L}\right)$, where $\tau_{i}=1$ if the site $i$ is occupied and $\tau_{i}=0$ otherwise. The probability $P_{t}(\mathcal{C})$ of being in con- 


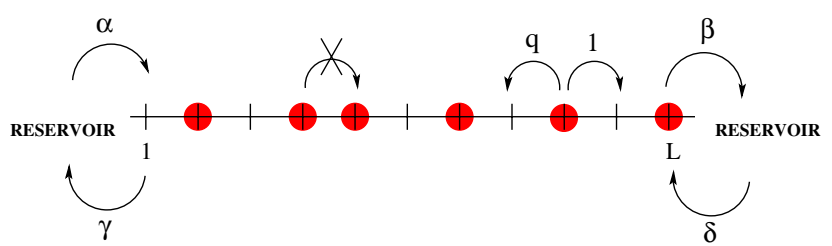

FIG. 1 (color online). Dynamical rules for the ASEP with open boundaries. The rate of forward jumps has been normalized to 1 . Backward jumps occur with rate $q<1$. All other parameters are arbitrary.

figuration $\mathcal{C}$ at time $t$ evolves according to the master equation

$$
\frac{d P_{t}(\mathcal{C})}{d t}=\sum_{\mathcal{C}^{\prime}} M\left(\mathcal{C}, \mathcal{C}^{\prime}\right) P_{t}\left(\mathcal{C}^{\prime}\right)
$$

The nondiagonal matrix element $M\left(\mathcal{C}, \mathcal{C}^{\prime}\right)$ represents the transition rate from $\mathcal{C}^{\prime}$ to $\mathcal{C}$. The diagonal element $M(\mathcal{C}, \mathcal{C})=-\sum_{\mathcal{C}^{\prime} \neq \mathcal{C}} M\left(\mathcal{C}^{\prime}, \mathcal{C}\right)$ is equal to minus the exit rate from $\mathcal{C}$

In the long time limit, the ASEP reaches a nonequilibrium steady state where each configuration $\mathcal{C}$ occurs with a probability $P^{\star}(\mathcal{C})$, that can be written as a matrix product [28]:

$$
P^{\star}(\mathcal{C})=\frac{1}{Z_{L}}\left\langle W\left|\prod_{i=1}^{L}\left[\tau_{i} D+\left(1-\tau_{i}\right) E\right]\right| V\right\rangle,
$$

where the operators $D$ and $E$, the bra vector $\langle W|$, and the ket vector $|V\rangle$ satisfy quadratic algebraic relations

$$
\begin{aligned}
D E-q E D & =(1-q)(D+E), \\
(\beta D-\delta E)|V\rangle & =(1-q)|V\rangle, \\
\langle W|(\alpha E-\gamma D) & =(1-q)\langle W| .
\end{aligned}
$$

The normalization constant in Eq. (2) is given by $Z_{L}=$ $\left\langle W\left|(D+E)^{L}\right| V\right\rangle$. The matrix product representation allows us to determine stationary equal-time correlations and density profiles for any system size $L$.

For $L \rightarrow \infty$, the ASEP has three phases whose boundaries are given in terms of the effective densities $\rho_{a}=$ $1 /\left(a_{+}+1\right)$ and $\rho_{b}=b_{+} /\left(b_{+}+1\right)$ of the left and right reservoirs, where

$$
\begin{aligned}
& a_{ \pm}=\frac{(1-q-\alpha+\gamma) \pm \sqrt{(1-q-\alpha+\gamma)^{2}+4 \alpha \gamma}}{2 \alpha}, \\
& b_{ \pm}=\frac{(1-q-\beta+\delta) \pm \sqrt{(1-q-\beta+\delta)^{2}+4 \beta \delta}}{2 \beta} .
\end{aligned}
$$

The ASEP is in the maximal current phase when $\rho_{a}>1 / 2$ and $\rho_{b}<1 / 2$, in the low density phase when $\rho_{a}<1 / 2$ and $\rho_{a}+\rho_{b}<1$, and in the high density phase when $\rho_{b}>1 / 2$ and $\rho_{a}+\rho_{b}>1$.
For a system of size $L$, the average value $J$ of the stationary current is given by the ratio $Z_{L-1} / Z_{L}$, which can be expressed in terms of orthogonal polynomials [34]. However, the fluctuations of the steady-state current cannot be calculated from the knowledge of the stationary probabilities alone. In order to study the current, we introduce an observable $Y_{t}$ that counts the number of particles exchanged between the system and the left reservoir between times 0 and $t$. Therefore, $Y_{t+d t}=Y_{t}+y$, where $y=+1$ if a particle enters the site $1, y=-1$ if a particle exits from 1 during the interval $d t$, and $y=0$ otherwise. These three mutually exclusive types of transitions lead to a three-part decomposition of the generator $M: M=M_{+}+M_{-}+M_{0}$. We note that $Y_{t}$ also represents the total integrated current that has flown through the system till time $t$. When $t \rightarrow \infty$, the expectation value of $Y_{t} / t$ converges to the average stationary current $J$. The convergence rate is quantified by the large-deviation function $\Phi(j)$, characterizing nontypical fluctuations of $Y_{t}$ and defined as $P\left(\frac{Y_{t}}{t}=j\right) \sim e^{-t \Phi(j)}$.

A different manner to encode the statistics of $Y_{t}$ is through its characteristic function which, in the long time limit, behaves as $\left\langle e^{\mu Y_{t}}\right\rangle \simeq e^{\mathcal{E}(\mu) t}$, where $\mathcal{E}(\mu)$ is the cumulant generating function of $Y_{t}$, and is the Legendre transform of the large-deviation function $\Phi(j)$ [14]: $\mathcal{E}(\mu)=\max _{j}[\mu j-\Phi(j)]$. Following Refs. [3,9], one can prove that $\mathcal{E}(\mu)$ is the largest eigenvalue of the deformed operator $M(\mu)=e^{\mu} M_{+}+e^{-\mu} M_{-}+M_{0}$. Thus, the calculation of the cumulants of the current is equivalent to an eigenvalue problem.

For the ASEP with periodic boundary conditions, $M(\mu)$ can be diagonalized by the Bethe ansatz, leading to a full solution for the current fluctuations [9,24]. In the case of open boundary conditions, integrability conditions are met only on hypersurfaces of the parameter space, and the Bethe ansatz can be used only for $L \rightarrow \infty$ and in specific regions of the phase diagram [30].

We have obtained a solution valid for all parameters and all system sizes using a generalized matrix product representation. The components $F_{\mu}(\mathcal{C})$ of the dominant eigenvector $F_{\mu}$ of $M(\mu)$ can be expanded formally as a power series with respect to $\mu$ to any given order $k \geq 0$. For each value of $k$, we have proved rigorously [36] that $F_{\mu}$ can be represented by a matrix product ansatz up to corrections of order $\mu^{k+1}$, i.e.,

$$
F_{\mu}(\mathcal{C})=\frac{1}{Z_{L}^{(k)}}\left\langle W_{k}\left|\prod_{i=1}^{L}\left[\tau_{i} D_{k}+\left(1-\tau_{i}\right) E_{k}\right]\right| V_{k}\right\rangle+\mathcal{O}\left(\mu^{k+1}\right)
$$

The matrices $D_{k}$ and $E_{k}$ are constructed recursively starting with $D_{1}=D$ and $E_{1}=E$ and

$$
\begin{aligned}
& D_{k+1}=(1 \otimes 1+d \otimes e) \otimes D_{k}+(1 \otimes d+d \otimes 1) \otimes E_{k}, \\
& E_{k+1}=(1 \otimes 1+e \otimes d) \otimes E_{k}+(e \otimes 1+1 \otimes e) \otimes D_{k},
\end{aligned}
$$


where we have defined the operators $d=D-1$ and $e=$ $E-1$ that satisfy the $q$-deformed harmonic oscillator algebra $d e-q e d=1-q$. These matrices are related to the ones used for the matrix product solution of the multispecies periodic ASEP [37].

The boundary vectors $\left\langle W_{k}\right|$ and $\left|V_{k}\right\rangle$ are constructed by taking tensor products of bra and ket vectors. We start with $\left|V_{1}\right\rangle=|V\rangle$ and $\left\langle W_{1}\right|=\langle W|$ and iterate

$$
\begin{gathered}
\left|V_{k+1}\right\rangle=|V\rangle \otimes|\tilde{V}\rangle \otimes\left|V_{k}\right\rangle, \\
\left\langle W_{k+1}\right|=\left\langle W^{\mu}\right| \otimes\left\langle\tilde{W}^{\mu}\right| \otimes\left\langle W_{k}\right|,
\end{gathered}
$$

where $|V\rangle$ is defined in Eq. (3) and

$$
\begin{gathered}
{[\beta(1-d)-\delta(1-e)]|\tilde{V}\rangle=0,} \\
\left\langle W^{\mu}\right|\left[\alpha\left(1+e^{\mu} e\right)-\gamma\left(1+e^{-\mu} d\right)\right]=(1-q)\left\langle W^{\mu}\right|, \\
\left\langle\tilde{W}^{\mu}\right|\left[\alpha\left(1-e^{\mu} e\right)-\gamma\left(1-e^{-\mu} d\right)\right]=0 .
\end{gathered}
$$

This matrix ansatz allows us to calculate the cumulants to any desired order $k$. Our central result is a parametric formula for the cumulant generating function $\mathcal{E}(\mu)$ :

$$
\mu=-\sum_{k \geq 1} C_{k} \frac{B^{k}}{k} \quad \text { and } \quad \mathcal{E}=-(1-q) \sum_{k \geq 1} D_{k} \frac{B^{k}}{k},
$$

where $B$ is a formal parameter that has to be eliminated from the two equations. We emphasize that similar parametric expressions have appeared in all known exact expressions for the current cumulant generating function $[9,24,38]$ and a similar generic form was derived from the additivity principle in Ref. [8]. The function $\mathcal{E}(\mu)$ is fully specified from the knowledge of the scalars $C_{k}$ and $D_{k}$. These are given by contour integrals in the complex plane along a contour $\Gamma$ (to be defined below):

$$
C_{k}=\oint_{\Gamma} \frac{d z}{2 i \pi} \frac{\phi_{k}(z)}{z}, \quad D_{k}=\oint_{\Gamma} \frac{d z}{2 i \pi} \frac{\phi_{k}(z)}{(z+1)^{2}} .
$$

The $\phi_{k}(z)$ 's are obtained as follows. We define a function $W_{B}(z)$ that depends on the parameter $B$ :

$$
W_{B}(z)=\sum_{k \geq 1} \phi_{k}(z) \frac{B^{k}}{k}
$$

and we find that $W_{B}(z)$ is uniquely determined as the solution of the functional equation:

$$
W_{B}(z)=-\frac{1}{2} \ln \left(1-B F(z) e^{X\left[W_{B}\right](z)}\right),
$$

where $F(z)$ is given by the expression

$$
\frac{(1+z)^{L}\left(1+z^{-1}\right)^{L}\left(z^{2}\right)_{\infty}\left(z^{-2}\right)_{\infty}}{\left(a_{+} z\right)_{\infty}\left(\frac{a_{+}}{z}\right)_{\infty}\left(a_{-} z\right)_{\infty}\left(\frac{a_{-}}{z}\right)_{\infty}\left(b_{+} z\right)_{\infty}\left(\frac{b_{+}}{z}\right)_{\infty}\left(b_{-} z\right)_{\infty}\left(\frac{b_{-}}{z}\right)_{\infty}}
$$

with $(x)_{\infty}=\prod_{k=0}^{\infty}\left(1-q^{k} x\right)$. We note that $F(z)$ appears in the definition of the Askey-Wilson polynomials, known to be relevant to the open ASEP [34,39]. The operator $X$ is a linear integral operator:

$$
X\left[W_{B}\right]\left(z_{1}\right)=\oint_{\Gamma} \frac{d z_{2}}{2 \imath \pi z_{2}} W_{B}\left(z_{2}\right) K\left(\frac{z_{1}}{z_{2}}\right),
$$

where the kernel $K$ is given by

$$
K(z)=2 \sum_{k=1}^{\infty} \frac{q^{k}}{1-q^{k}}\left\{z^{k}+z^{-k}\right\}
$$

and the contour $\Gamma$ in the complex plane encircles (once) the points $0, q^{k} a_{+}, q^{k} a_{-}, q^{k} b_{+}$, and $q^{k} b_{-}$for all integers $k \geq 0$. The kernel $K\left(z_{1} / z_{2}\right)$ was used in Ref. [24] to calculate the current fluctuations in the periodic case.

The functional equation (16) contains the complete information about the current statistics: By solving it iteratively to any order $k$, we obtain the first $k$ cumulants of the current. At first order, we have $\phi_{1}(z)=F(z) / 2$, and the mean value of the current is

$$
J=\lim _{t \rightarrow \infty} \frac{\left\langle Y_{t}\right\rangle}{t}=(1-q) \frac{D_{1}}{C_{1}}=(1-q) \frac{\oint_{\Gamma} \frac{d z}{2 i \pi} \frac{F(z)}{z}}{\oint_{\Gamma} \frac{d z}{2 i \pi} \frac{F(z)}{(z+1)^{2}}} .
$$

This expression is identical to that given in Ref. [34]. At second order, the variance of the current is

$$
\Delta=\lim _{t \rightarrow \infty} \frac{\left\langle Y_{t}^{2}\right\rangle-\left\langle Y_{t}\right\rangle^{2}}{t}=(1-q) \frac{D_{1} C_{2}-D_{2} C_{1}}{2 C_{1}^{3}},
$$

where $C_{2}$ and $D_{2}$ are obtained by using (14) with

$$
\phi_{2}(z)=\frac{1}{2}\left(F^{2}(z)+\oint_{\Gamma} \frac{d z_{2} F(z) F\left(z_{2}\right) K\left(z / z_{2}\right)}{2 \imath \pi z_{2}}\right) .
$$

For higher cumulants, exact expressions similar to Eq. (21) are obtained and can be expressed via a combinatorial tree expansion akin to that found in the periodic case [24]. The expression of the diffusion constant $\Delta$ generalizes the formula of Ref. [35] obtained for the totally asymmetric exclusion process (TASEP) in which $q=\delta=\gamma=0$. For the TASEP, the kernel $K$ and the operator $X$ vanish identically, and $F(z)$ reduces to

$$
F_{\mathrm{TASEP}}(z)=\frac{-(1+z)^{2 L}\left(1-z^{2}\right)^{2}}{z^{L}(1-a z)(z-a)(1-b z)(z-b)}
$$

with $a=\frac{1-\alpha}{\alpha}$ and $b=\frac{1-\beta}{\beta}$; then, Eq. (16) leads to $\phi_{k}(z)=$ $F_{\text {TASEP }}^{k}(z) / 2$, and the results of Ref. [38] are retrieved. For a periodic system of size $L$ with $N$ particles, the current fluctuations can be brought into the framework described here with the same generalized matrix ansatz, but the boundary vectors are replaced by a trace and Eq. (16) is modified as follows: The prefactor $1 / 2$ is removed, $F(z)=(1+z)^{L} / z^{N}$, and the kernel $K$ is still given by (19). Then, the results of Ref. [24], originally obtained by Bethe ansatz, are retrieved.

The derivation of the above results involves combinatorial identities for matrix elements of the generalized matrix ansatz. Some of these identities were guessed by induction rather than mathematically proved [36]. It was therefore necessary to validate our calculations numerically. For 
small size systems $(L \leq 7)$, expressions for the cumulants have been checked against the exact values from direct calculations. For larger systems $(L \leq 100)$, we compared the analytical formulas with numerical computations of the cumulants performed using a density matrix renormalization group (DMRG) method. That method, originally introduced to study ground state properties of quantum spin chains [40], was recently adapted to calculate the highest eigenvalues of deformed stochastic operators like $M(\mu)$ [11]. A few of those results are displayed in Figs. 2 and 3.

For large system sizes $L \rightarrow \infty$, the cumulant generating function takes different expressions in different phases. These are derived from an asymptotic analysis involving the leading singularities of $F(z)[29,34,36]$. In the low density phase, the dominant singularity is the pole at $a_{+}$ leading to $\phi_{k}(z) \sim F^{k}(z)$. Using the Lagrange inversion formula as in Ref. [38], we obtain

$$
\mathcal{E}(\mu)=(1-q)\left(1-\rho_{a}\right) \frac{e^{\mu}-1}{e^{\mu}+\left(1-\rho_{a}\right) / \rho_{a}} .
$$

This expression agrees with the one found in Ref. [30] using the Bethe ansatz. Its Legendre transform matches the prediction of the additivity principle [31]:

$$
\Phi(j)=(1-q)\left\{\rho_{a}-r+r(1-r) \ln \left(\frac{1-\rho_{a}}{\rho_{a}} \frac{r}{1-r}\right)\right\},
$$

where the current $j$ is parametrized as $j=(1-q) r(1-r)$. The high density phase leads to similar expressions with $a_{+} \rightarrow b_{+}$and $\rho_{a} \rightarrow 1-\rho_{b}$. In both cases, the statistics of the current do not depend on system size in the large $L$ limit.

In the maximal current phase, we find that $k$ th cumulant grows as $L^{(k-3) / 2}$. When $L \rightarrow \infty$, we have

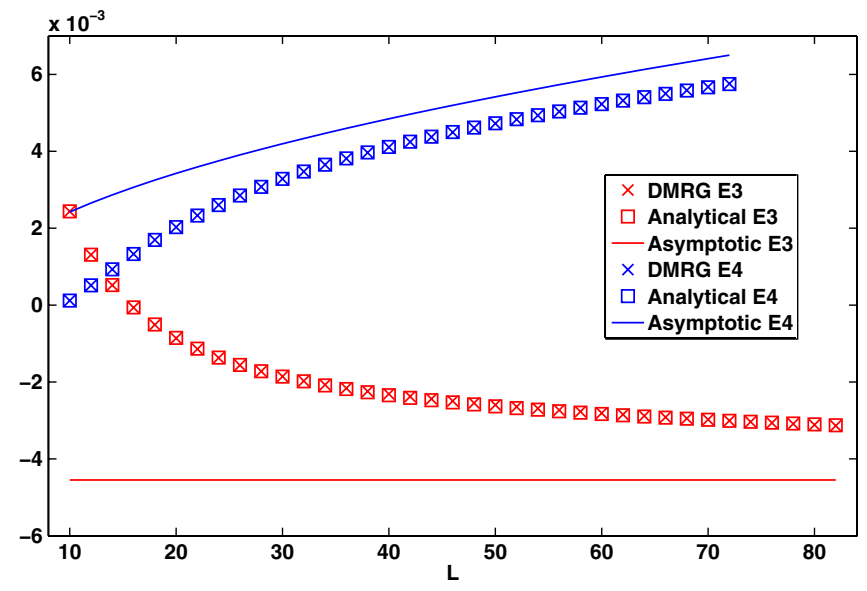

FIG. 2 (color online). Third (E3, red) and fourth (E4, blue) cumulants in the maximal current phase, with $q=0.5, a_{+}=$ $b_{+}=0.65$, and $a_{-}=b_{-}=0.6$; the full lines represent the corresponding large size asymptotic behaviors.

$$
\begin{gathered}
\mu=-\frac{L^{-1 / 2}}{2 \sqrt{\pi}} \sum_{k=1}^{\infty} \frac{(2 k) !}{k ! k^{(k+3 / 2)}} B^{k}, \\
\mathcal{E}-\frac{1-q}{4} \mu=-\frac{(1-q) L^{-3 / 2}}{16 \sqrt{\pi}} \sum_{k=1}^{\infty} \frac{(2 k) !}{k ! k^{(k+5 / 2)}} B^{k} .
\end{gathered}
$$

These expressions have the same structure as those obtained for the case of a periodic ring [9], and the largedeviation functions have the same asymptotic behavior. Moreover, for the open TASEP of size $L$ with $\alpha=1$ and $\beta=1 / 2$, we observed that the formulas are identical to those for the half-filled periodic TASEP of size $2 L+2$.

Along the shock line $\left(\rho_{a}=1-\rho_{b}<1 / 2\right)$, we obtain

$$
\begin{gathered}
\mu=-2 L^{-1} \frac{\left(1+a_{+}\right)}{\left(1-a_{+}\right)} \sum_{k=1}^{\infty} \frac{k^{2 k-1}}{(2 k) !} B^{k}, \\
\mathcal{E}-\frac{(1-q) a_{+}}{\left(1+a_{+}\right)^{2}} \mu=-2 L^{-2} \frac{(1-q) a_{+}}{\left(1-a_{+}^{2}\right)} \sum_{k=1}^{\infty} \frac{k^{2 k-2}}{(2 k) !} B^{k},
\end{gathered}
$$

with the $k$ th cumulant scaling as $L^{(k-2)}$ as can be explained by the domain wall picture for $\rho_{a} \ll 1[32,38]$. We note that this is the only case where the statistics of the current depend both on the system size and on the boundary parameters at the large $L$ limit.

We have obtained exact formulas for the current statistics of the open exclusion process in contact with two reservoirs. Our results are valid for arbitrary sizes and values of the parameters and have been tested by precise DMRG computations in various regions of the phase diagram. They could also be used as benchmarks to test alternative computational algorithms [12]. In the limit of large size systems, the asymptotic behavior of the largedeviation function is derived in all regions of the phase diagram as long as the asymmetry $(1-q)$ remains finite. The diffusive limit $q \rightarrow 1$ represents an important open analytical problem, and the exact formulas should coincide

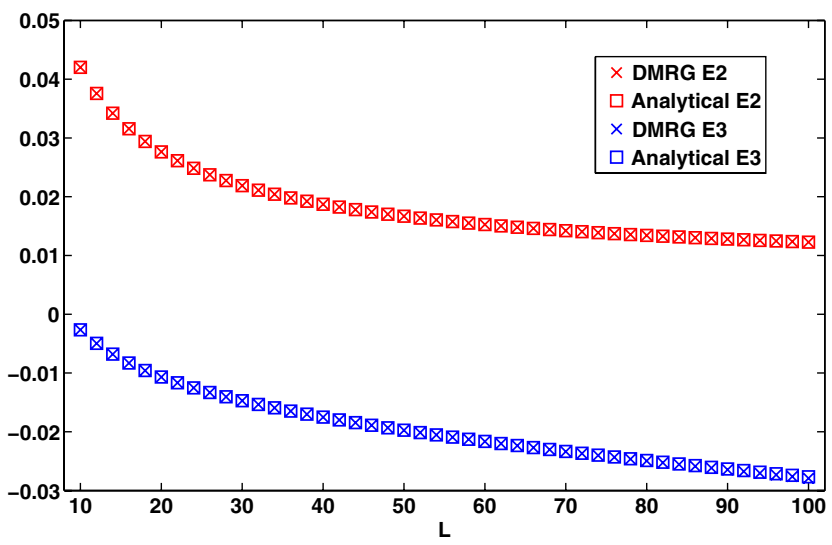

FIG. 3 (color online). Second (E2, red) and third (E3, blue) cumulants in the high density phase, with $q=0.5, a_{+}=0.28$, $b_{+}=1.15, a_{-}=-0.48$, and $b_{-}=-0.27$. 
with the predictions of macroscopic fluctuation theory [7,31]. We have used an extension of the matrix ansatz that was introduced for multispecies exclusion models [37]. The relation between multispecies models and current fluctuations (and also between open and periodic systems) is mysterious, as no direct mapping is known. We believe that our results should be derivable from the Bethe ansatz for a spin chain with nondiagonal boundaries, but the corresponding Bethe equations have not yet been derived [30]. In addition, the matrix representation given here contains all the information about the density profiles that generate atypical currents: The precise calculation of these profiles represents a challenging open question [1]. Finally, the effect of global constraints-such as a finite particle reservoir-on the current fluctuations is an interesting problem which requires further investigation [41].

A. L. thanks R. Vasseur for useful remarks. K. M. is thankful to S. Mallick for a very careful reading of the manuscript.

[1] B. Derrida, J. Stat. Mech. (2007) P07023; (2011) P01030.

[2] D. J. Evans, E. G. D. Cohen and G. P. Morriss, Phys. Rev. Lett. 71, 2401 (1993); G. Gallavotti and E. D. G. Cohen, Phys. Rev. Lett. 74, 2694 (1995); C. Jarzynski, Phys. Rev. Lett. 78, 2690 (1997).

[3] J. L. Lebowitz and H. Spohn, J. Stat. Phys. 95, 333 (1999).

[4] B. Schmittmann and R. K. P. Zia, in Phase Transitions and Critical Phenomena, edited by C. Domb and J.L. Lebowitz (Academic, San Diego, 1995), Vol. 17.

[5] G. M. Schütz, in Phase Transitions and Critical Phenomena, edited by C. Domb and J.L. Lebowitz (Academic, San Diego, 2001), Vol. 19.

[6] H. Spohn, Large Scale Dynamics of Interacting Particles (Springer-Verlag, New York, 1991).

[7] L. Bertini, A. De Sole, D. Gabrielli, G. Jona-Lasinio and C. Landim, Phys. Rev. Lett. 87, 040601 (2001); 94, 030601 (2005).

[8] T. Bodineau and B. Derrida, Phys. Rev. Lett. 92, 180601 (2004); Phys. Rev. E 72, 066110 (2005).

[9] B. Derrida and J. L. Lebowitz, Phys. Rev. Lett. 80, 209 (1998); B. Derrida and C. Appert, J. Stat. Phys. 94, 1 (1999); B. Derrida and M. R. Evans, J. Phys. A 32, 4833 (1999).

[10] B. Derrida, J. L. Lebowitz, and E. R. Speer, Phys. Rev. Lett. 89, 030601 (2002).

[11] M. Gorissen, J. Hooyberghs, and C. Vanderzande, Phys. Rev. E 79, 020101 (2009); M. Gorissen and C. Vanderzande, J. Phys. A 44, 115005 (2011).

[12] C. Giardina, J. Kurchan, and L. Peliti, Phys. Rev. Lett. 96, 120603 (2006); C. Giardina, J. Kurchan, V. Lecomte, and J. Tailleur, J. Stat. Phys. 145, 787 (2011); P. I. Hurtado and P. L. Garrido, Phys. Rev. Lett. 102, 250601 (2009).

[13] K. A. Takeuchi and M. Sano, Phys. Rev. Lett. 104, 230601 (2010).

[14] H. Touchette, Phys. Rep. 478, 1 (2009).

[15] B. Derrida, Phys. Rep. 301, 65 (1998).
[16] S. Katz, J. L. Lebowitz, and H. Spohn, J. Stat. Phys. 34, 497 (1984).

[17] S. R.S. Varadhan, in Itô Stochastic Calculus and Probability Theory (Springer, Tokyo, 1996) Vol. 385.

[18] T. Chou and D. Lohse, Phys. Rev. Lett. 82, 3552 (1999); T. Chou, K. Mallick, and R. K. P. Zia, Rep. Prog. Phys. 74, 116601 (2011).

[19] K. Johansson, Commun. Math. Phys. 209, 437 (2000); T. Kriecherbauer and J. Krug, J. Phys. A 43, 403001 (2010); I. Corwin, Random Matrices Theor. Appl. 1, 1130001 (2012).

[20] M. Kardar, G. Parisi and Y.-C. Zhang, Phys. Rev. Lett. 56, 889 (1986); T. Halpin-Healy, Y.-C. Zhang, Phys. Rep. 254, 215 (1995).

[21] J. Krug, Phys. Rev. Lett. 67, 1882 (1991).

[22] T. Sasamoto, J. Stat. Mech. (2007) P07007; T. Sasamoto and H. Spohn, Phys. Rev. Lett. 104, 230602 (2010); G. Amir, I. Corwin, and J. Quastel, Commun. Pure Appl. Math. 64, 466 (2011); P. L. Ferrari, J. Stat. Mech. (2010) P10016.

[23] D. Dhar, Phase Transit. 9, 51 (1987); L.-H. Gwa and H. Spohn, Phys. Rev. Lett. 68, 725 (1992); D. Kim, Phys. Rev. E 52, 3512 (1995); J. de Gier and F. H. L. Essler, Phys. Rev. Lett. 95, 240601 (2005).

[24] S. Prolhac, J. Phys. A 43, 105002 (2010).

[25] C. A. Tracy and H. Widom, Commun. Math. Phys. 279, 815 (2008); J. Math. Phys. (N.Y.) 50, 095204 (2009).

[26] G. M. Schütz, J. Stat. Phys. 88, 427 (1997); A. Rakos and G. M. Schütz, J. Stat. Phys. 118, 511 (2005).

[27] B. Derrida, E. Domany, and D. Mukamel, J. Stat. Phys. 69, 667 (1992); G. M. Schütz and E. Domany, J. Stat. Phys. 72, 277 (1993).

[28] B. Derrida, M. R. Evans, V. Hakim, and V. Pasquier, J. Phys. A 26, 1493 (1993)

[29] R. A. Blythe and M. R. Evans, J. Phys. A 40, R333 (2007).

[30] J. de Gier and F. H. L. Essler, Phys. Rev. Lett. 107, 010602 (2011).

[31] T. Bodineau and B. Derrida, J. Stat. Phys. 123, 277 (2006).

[32] M. Dudzinski and G. M. Schütz, J. Phys. A 33, 8351 (2000).

[33] B. Derrida, B. Douçot and P.-E. Roche, J. Stat. Phys. 115, 717 (2004).

[34] T. Sasamoto, J. Phys. A 32, 7109 (1999); M. Uchiyama, T. Sasamoto, and M. Wadati, J. Phys. A 37, 4985 (2004); M. Uchiyama and M. Wadati, J. Nonlinear Math. Phys. 12, 676 (2005).

[35] B. Derrida, M. R. Evans, and K. Mallick, J. Stat. Phys. 79, 833 (1995).

[36] A. Lazarescu et al. (to be published).

[37] S. Prolhac, M. R. Evans, and K. Mallick, J. Phys. A 42, 165004 (2009).

[38] A. Lazarescu and K. Mallick, J. Phys. A 44, 315001 (2011).

[39] S. Corteel and L. Williams, Duke Math. J. 159, 385 (2011).

[40] S. R. White, Phys. Rev. Lett. 69, 2863 (1992); Phys. Rep. 301, 187 (1998); U. Schollwöck, Ann. Phys. (N.Y.) 326, 96 (2011).

[41] D. A. Adams, B. Schmittmann, and R. K. P. Zia, J. Stat. Mech. (2008), P06009. 